\title{
A Per Model of Polymorphism and Recursive Types
}

\author{
M. Abadi* $\quad$ G. D. Plotkin ${ }^{\dagger}$
}

\section{Synopsis}

A model of Reynolds' polymorphic lambda calculus is provided, which also allows the recursive definition of elements and types. The technique is to use a good class of partial equivalence relations over a certain cpo. This allows the combination of inverse-limits for recursion and intersection for polymorphism.

\section{Introduction}

The ideal model provides an interpretation for a rich type system, with polymorphism and recursion [12], but not a model of the typed $\lambda$-calculus. In search for a satisfactory semantics for $\lambda$-calculi with recursive and polymorphic types, it seems natural, then, to consider partial equivalence relations (pers) instead of ideals. As ideals are certain subsets of a universal domain $D$, we replace them with certain pers over $D$ (rather than over $\omega$, as in $[8,19])$. For example, in order to interpret recursion, only the pers over $D$ that satisfy a completeness axiom should be considered.

This study was begun by Amadio and Cardone $[1,5]$. They left open how to find complete partial orders on pers so that recursive types could be obtained by applying the usual inverselimit construction [18]. In the case of models

\footnotetext{
${ }^{*}$ Digital Equipment Corporation, Systems Research Center, 130 Lytton Avenue, Palo Alto, California 94301, U.S.A.

${ }^{\dagger}$ Dept. of Computer Science, University of Edinburgh, King's Buildings, Edinburgh EH9 3JZ, U.K. (Part of this work was completed while at Digital Equipment Corporation, Systems Research Center.)
}

along the lines of Girard (e.g., see [7]) recursive definitions of elements and types are already available.

We define a class of "good" pers yielding complete partial orders. The partial orders obtained are intrinsic (and there are also intrinsic topologies). The corresponding idea for pers over $\omega$ is considered by many authors. A general setting is that of Rosolini: a topos with a dominance [15]. Scott has advocated developing the theory in the internal logic; we have yet to attempt this for good pers over $D$-one feature is that it is only $\neg \neg$ true that they are cpos.

We provide a model of an extension of $\lambda_{2}$, Reynolds' polymorphic $\lambda$-calculus. Taking $\lambda_{2}$ as in [3], say, we add the type expression $(\mu X: T p . T)$ for recursive types, the term expressions $(\mu x: T . e)$, for recursive elements, and $\eta X: T p . T$ and $\eta^{-1} X: T p . T$ for an isomorphism $\eta X: T p \cdot T: T[\mu X: T p \cdot T / X] \cong \mu X: T p \cdot T$ and its inverse (see also [6]).

The semantics is presented as a Seely model $[3$, 17] with extra structure for recursion. We omit the evident interpretation of the new syntactical forms. To the usual equations one adds $e[\mu x: T . e / x]=\mu x: T . e$ and two more expressing the isomorphism.

We represent Amadio's use of metric-space methods as another model of the same kind; it turns out that the two are essentially identical. However, while one can easily extend the metric model to deal with subtypes, that does not seem to be the case for the order-theoretic model.

The axioms for good pers are somewhat restrictive, and force the intrinsic partial orders to 
be consistently complete; this has the disadvantage of excluding the convex powerdomain as a type constructor. We consider partial preorders, which are pers equipped with a partial order on their field. We impose weaker axioms (just completeness and uniformity - see below) and obtain yet another model of the extended $\lambda_{2}$, but now also allowing the convex powerdomain as a type constructor.

We would like to extend the order-theoretic approach further; it seems possible to model the theory of constructions. Our work began as an attempt to build on the work of Cardelli and Longo [4] to model the language Quest, with its records, subtypes, and powertypes. While records produce no new problems, and the traditional view of subtypes as subpers goes a long way, the combination of recursive types and polymorphism with bounded quantifiers has defeated us. The difficulty is caused by the possibility of a type recursion going through a bound.

In the next section we describe the cpo that serves as setting for this work. We study partial equivalence relations in section 4 . In section 5 we define type constructions in an $\mathbf{O}$-category of pers. In sections 6 and 7 we give an interpretation of types and terms; section 8 describes the alternative metric approach. We move from pers to partial preorders in section 9. We assume standard domain-theoretic and categorical concepts and notation $[9,12,18]$.

\section{Background Assumptions}

We work with the partial equivalence relations over a cpo $D$ such that:

1. There is an increasing sequence $\pi_{n}: D \rightarrow D$ of continuous projections with least upper bound the identity and with finite range. Further, $\pi_{0}=\perp$.

2. There are strict, continuous retraction pairs:

$$
\begin{array}{rll}
\mathscr{D} \stackrel{e_{\mathscr{O}}}{\longrightarrow} & D & \stackrel{r_{\mathbb{P}}}{\longrightarrow} \mathbb{D} \\
\mathbb{N} \stackrel{e_{\mathbb{N}}}{\longrightarrow} & D & \stackrel{r_{\mathbb{N}}}{\longrightarrow} \mathbb{N} \\
D \times D \stackrel{e_{\times}}{\longrightarrow} & D & \stackrel{r_{\times}}{\longrightarrow} D \times D \\
D \rightarrow D \stackrel{e_{\rightarrow}}{\longrightarrow} & D & \stackrel{r_{\rightarrow}}{\longrightarrow} D \rightarrow D
\end{array}
$$

Here $\mathscr{O}$ is the two-point cpo $\{\perp, *\}$ and $\mathbb{N}$ is that of the natural numbers.

3. For all $n$, the following hold:

$$
\begin{aligned}
\pi_{n+1}\left(e_{\mathscr{O}}(*)\right) & \in e_{\mathscr{O}}(\mathbb{D}) \\
\pi_{n+1}\left(e_{\mathbb{N}}(m)\right) & \in e_{\mathbb{N}}(\mathbb{N}) \\
\pi_{n+1}\left(e_{\times}(\langle x, y\rangle)\right) & =e_{\times}\left(\left\langle\pi_{n}(x), \pi_{n}(y)\right\rangle\right) \\
\pi_{n+1}\left(e_{\rightarrow}(f)\right) & =e_{\rightarrow}\left(\pi_{n} \circ f \circ \pi_{n}\right)
\end{aligned}
$$

4. Meet closure holds, meaning that $D$ has all non-empty meets, that these are preserved by the $\pi_{n}$, and that all the $e$ 's and $r$ 's preserve these meets.

Below we omit to write the $e$ 's or $r$ 's and because of 2 allow ourselves the untyped $\lambda$-calculus as a notation for elements of $D$. Note that $1 \mathrm{im}-$ plies $\pi_{m} \circ \pi_{n}=\pi_{k}$ where $k=\min (m, n)$. Assumption 1 is equivalent to saying that $D$ is bifinite; in particular $\pi_{n}(x)$ is always finite. With 4 one has that $D$ is a Scott domain.

To obtain $D$ one can solve an appropriate domain equation, such as:

$$
D \cong \mathbb{O}+\mathbb{N}+(D \times D)+(D \rightarrow D)
$$

by the usual "limit of a sequence of iterates" process (see [18]) and obtain each $\pi_{n}$ by modifying the maps to the $n$-th iterate to ensure that $\pi_{n}\left(e_{\mathbb{N}}(\mathbb{N})\right)$ is finite.

\section{Partial Equivalence Relations}

First, some notation. If $S$ is a per then $|S|$ is the set $\{x \in D \mid x S x\}$; if $x$ is in $|S|$ then $[x]_{S}$ is its equivalence class; $[S]$ is the set of equivalence classes. If $f$ is in $(D \rightarrow D)$ and $S$ and $T$ are pers then we write $f: T^{S}$ to mean that $x S y$ implies $(f x) T(f y)$ for all $x$ and $y$. We associate to a subset $X \subset D$ the per $\{\langle x, x\rangle \mid x \in X\}$ and may write it as $X$.

The partial equivalence relations form a category Per with morphisms the set-theoretic functions $g:[S] \rightarrow[T]$ such that, for some $f: T^{S}$, if $x \in|S|$ then $g\left([x]_{S}\right)=[f(x)]_{T}$. We write $[f]_{S, T}$ for $g$ as $f$ uniquely determines it; we also write $f \vdash g$ (read $f$ realises $g$ ) if $g=[f]_{S, T}$. Per 
is effective (we should say $D$-effective) in that, first, there exists an element comp of $D$ (it is $\lambda p \lambda x . f s t(p)(\operatorname{snd}(p)(x)))$ such that if $f \vdash g$ and $f^{\prime} \vdash g^{\prime}$ then $\operatorname{comp}\left(\left\langle f, f^{\prime}\right\rangle\right) \vdash g \circ g^{\prime}$ (for composable $\left.g, g^{\prime}\right)$, and second, there is an element $\lambda x . x$ realising every identity $i d_{S}$.

As pointed out by Amadio and Cardone, one needs a completeness axiom on pers to model recursion $[1,5]$ :

Completeness $\perp S \perp$ and whenever $R \subset S$ is directed as a subset of $D^{2}$ then $\sqcup R$ is in $S$.

It is natural in domain theory to consider axioms relating infinite elements to finite ones. So one might try the assumption that if $x S y$ then there are $a_{n} S b_{n}$ with the $a_{n}, b_{n}$ finite, $x=\sqcup a_{n}$, and $y=\sqcup b_{n}$. Unfortunately the class of these pers (even the complete ones) is not closed under intersection. However, the following property introduced by Amadio and Cardone does yield closure:

Uniformity If $x S y$ then $\pi_{n}(x) S \pi_{n}(y)$ for all $n$.

They termed this property algebraicity or closure under approximation, but we prefer our term as the finite elements are found in a uniform way for all such pers. Note that uniformity can be written as $\pi_{n}: S^{S}$.

An intrinsic preorder can be defined on any per by putting, for all $x, y \in|S|$,

$$
x \leq_{S} y \text { iff } \forall f: \mathbb{O}^{S} \cdot(f(x)=* \supset f(y)=*)
$$

This defines a complete preorder on $|S|$ including $\sqsubseteq$ and $S$ (since the functions considered are continuous, monotone, and respect $S$ ). Assuming $S$ is complete and uniform, we can reverse this. Let $\prec_{S}$ be the least preorder on $|S|$ containing $\left.\sqsubseteq|| S\right|^{2}$ and $S$.

Lemma 1 1. If $a$ is finite then there is an $f: \mathbb{D}^{S}$ such that for any $y$ in $|S|, a \prec_{S} y$ iff $f(y)=*$.

2. For any $y$ and finite $a, a \leq_{S} y$ iff $a \prec_{S} y$.

\section{Proof}

1. Set $f(y)$ to be $*$ if $a \prec_{S} z \sqsubseteq y$ (for some $z$ ) and $\perp$ otherwise. That $f$ is monotone and respects the per is evident. For continuity, suppose $\left\{y_{\lambda}\right\}$ is directed and $f\left(\sqcup y_{\lambda}\right)=*$. Then $a \prec_{S} z \sqsubseteq \sqcup y_{\lambda}$ (for some $z$ ). Now $\pi_{n}(a)=a$ for some $n$, and then, since $S$ is uniform, $a=\pi_{n}(a) \prec_{S} z \sqsubseteq$ $\sqcup \pi_{n}\left(y_{\lambda}\right)=$ some $\pi_{n}\left(y_{\lambda}\right)$ as $\pi_{n}$ has finite range. But then $a \prec_{S} z \sqsubseteq \pi_{n}\left(y_{\lambda}\right) \sqsubseteq y_{\lambda}$, so $f\left(y_{\lambda}\right)=*$.

2. Follows from part 1 immediately.

It follows at once from part 2 of Lemma 1 that $\leq_{S}$ is the least complete preorder containing $\sqsubseteq$ and $S$.

For the rest of this section, only complete, uniform pers are considered.

To work with a partial order on $[S]$ we use:

Antisymmetry If $x \leq_{S} y \leq_{S} x$ then $x S y$.

For $x$ and $y$ in $|S|$, let $[x]_{S} \leq[y]_{S}$ iff $x \leq_{S} y$, obtaining a well-defined partial order.

Theorem 1 Suppose that $S$ is antisymmetric. When partially ordered by $\leq,[S]$ is an $\omega$-algebraic cpo. Its least element is $[\perp]_{S}$; if $x_{\lambda}$ is an $\sqsubseteq$-directed set in $|S|$ then $\left[\sqcup x_{\lambda}\right]_{S}$ is the $\leq$-lub of the $\left[x_{\lambda}\right]_{S}$. The finite elements are the $[a]_{S}$ with a finite and in $|S|$.

Proof First, $[\perp]_{S} \leq[x]_{S}$ for all $x$ in $|S|$, because $\leq_{S}$ contains $\left.\sqsubseteq\right|_{|S|^{2}}$. For completeness, let $\left[x_{\lambda}\right]_{S}$ be a directed family; let

$$
I_{n}=\left\{a \mid \forall \lambda \exists \lambda^{\prime} \geq \lambda . \pi_{n}\left(x_{\lambda^{\prime}}\right)=a\right\}
$$

Then $I_{n}$ is finite and non-empty and each element in $I_{n}$ is $\sqsubseteq$ an element in $I_{n+1}$. So there is a sequence $b_{n} \in I_{n}$ with $b_{n} \sqsubseteq b_{n+1}$. Then $\left[\bigsqcup_{n \geq 0} b_{n}\right]_{S}$ is the lub of the $\left[x_{\lambda}\right]_{S}$. That $\left[\sqcup x_{\lambda}\right]_{S}$ is the $\leq$-lub is because $\leq_{S}$ contains $\left.\sqsubseteq\right|_{|S|^{2}}$ and $S$ and is complete. To see that $[a]_{S}$ is finite, suppose $[a]_{S} \leq$ the lub of $\left\{\left[x_{\lambda}\right]_{S}\right\}$. Then $a \prec_{S} \sqcup b_{n}$ with the above notation, so, as in the proof of Lemma $1, a \prec_{S}$ some $b_{n}$ and, as $b_{n} \in I_{n},[a]_{S} \leq$ some $\left[x_{\lambda}\right]_{S}$. Then one completes the proof by noting that every $[x]_{S}$ is the $\leq$-lub of the $\left[\pi_{n}(x)\right]_{S}$.

We will write $\bigvee$ for lubs in $\langle[S], \leq\rangle$.

There is also an intrinsic topology.

Definition $1 V \subset[S]$ is open in the intrinsic topology iff $V=\left\{[x]_{S} \mid f(x)=*\right\}$ for some $f: \mathbb{D}^{S}$. 
Clearly, if $g:[S] \rightarrow[T]$ then $g$ is continuous with respect to the intrinsic topology.

Theorem 2 Suppose $S$ is antisymmetric. The intrinsic topology is the Scott topology.

Proof Set $V_{a}=\left\{[x]_{S} \mid a \leq_{S} x\right\}$. By Lemma 1, $V_{a}$ is open in the intrinsic topology. But by Theorem 1, every Scott open is a union of $V_{a}$ 's, and so also open in the intrinsic topology. For the converse, one shows that if $V$ is open in the intrinsic topology then $V=\cup\left\{V_{a} \mid[a]_{S} \in V\right\}$.

It follows that any $g:[S] \rightarrow[T]$ is continuous in that it preserves $\leq$-directed lubs.

In order to relate the intrinsic order on a per constructed from other pers to the intrinsic order on the latter we use other properties, and particular a meet-closure property. These properties are not actually necessary to our constructions, but they help one's understanding, and they would play a role in an inequational logic for $\lambda_{2}$.

Meet Closure of Pers Given non-empty families $x_{\lambda}$ and $y_{\lambda}$, if $x_{\lambda} S y_{\lambda}$ for every $\lambda$ then $\left(\sqcap_{\lambda} x_{\lambda}\right) S\left(\sqcap_{\lambda} y_{\lambda}\right)$.

The meet-closure condition resembles one suggested by Scott [16], where $x_{\lambda}$ is kept constant. It can be shown that the meet-closure property follows from its restriction to finite families.

It is desirable to avoid this meet-closure property for then we can work with bifinite cpos, and add powerdomains. One way of achieving this is to move from partial equivalence relations to partial preorders, as we do in section 9 .

If $S$ is meet closed, every equivalence class $[x]_{S}$ has a least element $\mu_{S}(x)=\sqcap\{y \mid y S x\}$.

Lemma 2 1. For $x, y \in|S|$, if $x \sqsubseteq y$ then $\mu_{S}(x) \sqsubseteq \mu_{S}(y)$.

2. For $x$ in $|S|$,

$$
\mu_{S}(x)=\bigsqcup\left\{\mu_{S}(a)|a \in| S \mid, a \sqsubseteq x, a \text { finite }\right\}
$$

3. For $x, y \in|S|, x \leq_{S} y$ iff $\mu_{S}(x) \sqsubseteq \mu_{S}(y)$.

\section{Proof}

1. As $\mu_{S}(x) S x$ and $\mu_{S}(y) S y$, we obtain also $\left(\mu_{S}(x) \sqcap \mu_{S}(y)\right) S(x \sqcap y)=x$. So $\mu_{S}(x) \sqsubseteq$ $\left(\mu_{S}(x) \sqcap \mu_{S}(y)\right) \sqsubseteq \mu_{S}(y)$.

2. By part $1, \mu_{S}(x) \sqsupseteq$ the lub. As we have $\mu_{S}\left(\pi_{n}(x)\right) S \pi_{n}(x)$, we can use the completeness of $S$ to get $\bigsqcup_{n} \mu_{S}\left(\pi_{n}(x)\right) S x$ and so $\mu_{S}(x) \sqsubseteq$ the lub.

3. Suppose $x \leq_{S} y$. If $a$ is finite in $|S|$ and $\sqsubseteq x$ then $a \prec_{S} y$ so $\mu_{S}(a) \sqsubseteq \mu_{S}(y)$ by part 1 . Then $\mu_{S}(x) \sqsubseteq \mu_{S}(y)$ by part 2 . The converse is trivial.

Part 3 shows that for such $S$, the intrinsic order coincides with one of the preorders considered by Amadio in [1] and also that completeness, uniformity, and meet closure imply antisymmetry.

Now set

$$
\pi_{S}(x)=\bigsqcup\left\{\mu_{S}(a)|a \in| S \mid, a \sqsubseteq x, a \text { finite }\right\}
$$

for all $x$ in $D$. The lub exists by consistent completeness

Theorem 3 1. $\pi_{S}$ is a continuous projection extending $\mu_{S}$.

2. If $x, y \in|S|$ then $x \leq_{S} y$ iff $\pi_{S}(x) \sqsubseteq \pi_{S}(y)$ iff $\exists z . x S z \sqsubseteq y$.

3. If $\left[x_{\lambda}\right]_{S}$ is $\leq$-consistent then

$$
\bigvee_{\lambda}\left[x_{\lambda}\right]_{S}=\left[\bigsqcup_{\lambda} \pi_{S}\left(x_{\lambda}\right)\right]_{S}
$$

4. For any non-empty family $x_{\lambda}$ in $|S|$,

$$
\bigwedge_{\lambda}\left[x_{\lambda}\right]_{S}=\left[\sqcap_{\lambda} \pi_{S}\left(x_{\lambda}\right)\right]_{S}
$$

Proof Omitted.

The last property we shall consider says that the equivalence classes are convex:

Convexity If $x \sqsubseteq y \sqsubseteq z$ and $x S z$ then $x S y$.

A per is good iff it is complete, uniform, meet closed, and convex. 


\section{Constructions and an O-Category of Pers}

We need a variety of constructions to build up types. To start we have $\mathbb{O}$ and $\mathbb{I N}$; then we need products and function spaces to get simple types; then we need intersections to get polymorphism; then finally we need certain inverse limits to interpret recursive equations for types. The inverse limits are taken in an $\mathbf{O}$-category of good pers, available once we have considered function spaces.

\section{Basic types}

The pers $\mathbb{O}$ and $\mathbb{N}$ are good, and the intrinsic order on them is $\sqsubseteq$.

\section{Cartesian closure}

As is well-known, Per is Cartesian closed. As terminal object we can take $\mathbb{1}=\{\langle\perp, \perp\rangle\}$, and the unique $S \rightarrow \mathbb{1}$ is $[\lambda x \cdot \perp]_{S, \mathbb{1}}$. For products take

$$
S \times T=\left\{\left\langle\langle x, y\rangle,\left\langle x^{\prime}, y^{\prime}\right\rangle\right\rangle \mid x S x^{\prime}, y T y^{\prime}\right\}
$$

The two projections are $[\lambda x . f s t(x)]_{S \times T, S}$ and $[\lambda x . s n d(x)]_{S \times T, T}$, and the pairing of $[f]_{R, S}$ and $[g]_{R, T}$ is $[\lambda x .\langle f(x), g(x)\rangle]_{R, S \times T}$. For function spaces take

$$
T^{S}=\left\{\langle f, g\rangle\left|f, g: T^{S}, \forall x \in\right| S \mid \cdot(f x) T(g x)\right\}
$$

Evaluation is $[\lambda w \cdot f s t(w)(\operatorname{snd}(w))]_{T^{S} \times S, T}$ and the currying of $[f]_{R \times S, T}$ is $[\lambda x . \lambda y . f(\langle x, y\rangle)]_{R, T^{S}}$. Note that we have a bijection $\phi:\left[T^{S}\right] \cong$ $\operatorname{Per}(S, T)$ where $\phi\left([f]_{T^{S}}\right)=[f]_{S, T}$.

Let us see how these constructions preserve the axioms. First $\mathbb{1}$ is good. Next $S \times T$ and $T^{S}$ are complete and uniform if $S$ and $T$ are [1].

Proposition 1 1. $S \times T$ and $T^{S}$ are meetclosed if $S$ and $T$ are.

2. $S \times T$ and $T^{S}$ are convex if $S$ and $T$ are.
Proof We just consider function spaces. For part 1 , one uses the fact that for any family $f_{\lambda}$ in $(D \rightarrow D)$,

$$
\left(\sqcap f_{\lambda}\right)(x)=\bigsqcup\left\{\sqcap_{\lambda} f_{\lambda}(a) \mid a \sqsubseteq x, a \text { finite }\right\}
$$

For part 2, suppose $T$ convex, $f \sqsubseteq g \sqsubseteq h$, and $f\left(T^{S}\right) h$. Take $x$ in $|S|$. Then $f x T h x$ and $f x \sqsubseteq$ $g x \sqsubseteq h x$. So $g x T f x$ showing $g T^{S} f$, as $f$ is in $\left|T^{S}\right|$.

The induced order on $\mathbb{1}$ is the evident $\perp \leq_{\mathbb{1}} \perp$.

Proposition 2 1. $\langle x, y\rangle \leq_{S \times T}\left\langle x^{\prime}, y^{\prime}\right\rangle$ iff both $x \leq_{S} x^{\prime}$ and $y \leq_{T} y^{\prime}$.

2. If $f \leq_{T^{S}} g$ then for any $x$ in $|S|, f x \leq_{T} g x$. The converse holds if $S$ is complete, uniform, and meet closed.

\section{Proof}

1. The implication from left to right holds as the projections are monotone. The implication from right to left holds as $f=[\lambda z \cdot\langle z, y\rangle]_{S, S \times T}$ and $g=\left[\lambda z \cdot\left\langle x^{\prime}, z\right\rangle\right]_{T, S \times T}$ are monotone and so: $[\langle x, y\rangle]_{S \times T}=f\left([x]_{S}\right) \leq f\left(\left[x^{\prime}\right]_{S}\right)=\left[\left\langle x^{\prime}, y\right\rangle\right]_{S \times T} \leq$ $\left[\left\langle x^{\prime}, y^{\prime}\right\rangle\right]_{S \times T}$, similarly. (This idea is the invention of Paul Taylor.)

2. The implication from left to right holds as the function $[\lambda h . h(x)]_{T^{S}, S}$ is monotone. In the other direction we have for $x$ in $|S|,\left(\pi_{T} \circ f\right) x \sqsubseteq g x$, and $f T^{S}\left(\pi_{T} \circ f\right)$, so $f T^{S}\left(\pi_{T} \circ f\right) \sqsubseteq g$.

\section{Intersection}

Amadio showed the uniform complete pers closed under intersection. The preservation of meet closure and convexity are both easy.

Proposition 3 Let $S_{\lambda}$ be a family of complete uniform pers and set $S=\cap S_{\lambda}$. If $x \leq_{S} y$ then $x \leq_{S_{\lambda}} y$. The converse holds if the $S_{\lambda}$ are also meet closed and convex.

Proof The implication from left to right holds by the monotonicity of $[\lambda x . x]_{S, S_{\lambda}}$. Conversely assume $x \leq_{S_{\lambda}} y$. Then $\pi_{S_{\lambda}} x \sqsubseteq y$. Set $z=$ $\bigsqcup_{\lambda} \pi_{S_{\lambda}} x$. Then as $\pi_{S_{\lambda}} x \sqsubseteq z \sqsubseteq x$ we get $x S z$. But $z \sqsubseteq y$ so $x \leq_{S} y$. 


\section{An O-Category of Pers}

Take the full subcategory $\mathbf{Q}$ of Per consisting of the good pers. The morphisms are partially ordered by $\leq$, where for $g, h: S \rightarrow T$

$$
g \leq h \text { iff } \forall x \in|S| . g\left([x]_{S}\right) \leq h\left([x]_{S}\right)
$$

With this, the bijection from $\left[T^{S}\right]$ to $\mathbf{Q}(S, T)$ is an isomorphism of partial orders by Proposition 2. So we obtain an $\mathbf{O}$-category in the sense of [18], that is, there is a least element, $[\lambda x . \perp]_{S, T}$, lubs of chains (even all directed sets) exist, and are defined pointwise, composition is continuous and left-strict.

To get uniformity preserved by the limits used to solve domain equations we consider uniform morphisms, those $g: S \rightarrow T$ such that $g \circ\left[\pi_{n}\right]_{S, S}=\left[\pi_{n}\right]_{T, T} \circ g$. Note that all uniform morphisms are strict.

To get meet closure preserved we consider the multiplicative morphisms, those preserving meets of non-empty families. This is equivalent to preserving binary meets, for uniform morphisms. The combination of uniformity and multiplicativity is preserved by products and function spaces.

Take $\mathbf{R}$ to be the subcategory of $\mathbf{Q}$ of all uniform multiplicative morphisms; it is a sub-Ocategory of $\mathbf{Q}$. Also $\mathbf{R}^{D}$ is the category with the same objects as $\mathbf{R}$ and as morphisms pairs $S \stackrel{f}{\longrightarrow} T \stackrel{g}{\longrightarrow} S$. It inherits an O-category structure from $\mathbf{R}$ by: $\langle f, g\rangle \leq\left\langle f^{\prime}, g^{\prime}\right\rangle$ iff $f \leq f^{\prime}$ and $g \leq g^{\prime}$. We set $\langle f, g\rangle^{\dagger}=\langle g, f\rangle$.

\section{Colimits}

We construct colimits in $\mathbf{R}^{D}$ of certain $\omega$-cochains of embedding-projection pairs. Let $\triangle=$ $\left\langle S_{m},\left\langle f_{m n}, g_{m n}\right\rangle\right\rangle$ be a cochain of embeddingprojection pairs. Suppose too that $\alpha_{m} \vdash f_{m n}$ and $\alpha_{m} \vdash g_{m n}$ for all $m, n \geq 0$ and also that $\alpha_{m} \sqsubseteq \alpha_{m+1}$ for $m \geq 0$.

Define $S_{\infty}$ by: $x S_{\infty} y$ iff $\forall n \forall_{\infty} m . \pi_{n} x S_{m} \pi_{n} y$ (here $\forall_{\infty}$ means "for almost all"). Then $S_{\infty}$ is a good per and $\alpha_{m}$ realises $\mathbf{R}$ morphisms $i_{m}: S_{m} \rightarrow S_{\infty}$ and $j_{m}: S_{\infty} \rightarrow S_{m}$. Further $\left\langle i_{m}, j_{m}\right\rangle$ is a cone of embedding-projection pairs from $\triangle$ to $S_{\infty}$ such that $i_{m} \circ j_{m}$ is increasing and has lub the identity on $S_{\infty}$. So, much as in [18], $\left\langle i_{m}, j_{m}\right\rangle: \triangle \rightarrow S_{\infty}$ is colimiting in $\mathbf{R}^{D}$. As regards the intrinsic order one has that $x \leq_{S_{\infty}} y$ iff $\forall m . \alpha_{m}(x) \leq_{S_{m}} \alpha_{m}(y)$ iff $\forall n \forall_{\infty} m . \pi_{n} x \leq_{S_{m}} \pi_{n} y$.

\section{$6 \quad$ Interpreting Types}

In the usual Per-based models of $\lambda_{2}$, one interprets types as functions over the set of pers and universal quantification as intersection. We wish to use the partial order structure on morphisms to solve recursive domain equations and so one needs a category, perhaps that of embeddingprojection pairs in $\mathbf{R}$, following [18]. To make intersection yield a functor one then needs to have functors be effective. But then one can generalise to the category $\mathbf{R}^{D}$ of all pairs of morphisms. The advantage of this is that the effective functors are automatically locally continuous in the sense of [18].

Let us say that $x \vdash\langle f, g\rangle$ when $x$ is $\langle y, z\rangle$ and $y \vdash f$ and $z \vdash g$. A functor $F:\left(\mathbf{R}^{D}\right)^{m} \rightarrow\left(\mathbf{R}^{D}\right)^{n}$ is effective iff there are $\phi_{j}(j=1, n)$ in $D$ such that if $x_{i} \vdash h_{i}(i=1, m)$ then $\phi_{j}(\hat{x}) \vdash$ $F(\hat{h})_{j}(j=1, n)$, using an evident vector notation. (We say $\hat{\phi}$ realises $F$.) Note that effectiveness is a condition on the behaviour of a functor on arrows. Local continuity then arises from the continuity of the realisers. We say the functor is symmetric iff $F\left(h_{1}^{\dagger}, \ldots, h_{m}^{\dagger}\right)_{j}=$ $\left(F\left(h_{1}, \ldots, h_{m}\right)_{j}\right)^{\dagger}(j=1, m)$. Then we say $\hat{\phi}$ realises $F$ symmetrically if $\phi_{j}\left(x_{1}, \ldots, x_{m}\right)^{\star}=$ $\phi_{j}\left(x_{1}^{\star}, \ldots, x_{m}^{\star}\right)$, where $\star=\lambda x \cdot\langle\operatorname{snd}(x), f s t(x)\rangle$ (for $j=1, n$ ). If $\hat{\phi}$ realises a symmetric $F$ then $\hat{\psi}$ realises $F$ symmetrically if $\psi_{j}\left(x_{1}, \ldots, x_{m}\right)$ equals $\left\langle f s t\left(\phi_{j}\left(x_{1}, \ldots, x_{m}\right)\right), f s t\left(\phi_{j}\left(x_{1}^{\star}, \ldots, x_{m}^{\star}\right)\right)\right\rangle$. Symmetric (covariant) functors on $\mathbf{R}^{D}$ arise naturally from functors on $\mathbf{R}$ which are covariant in some arguments and contravariant in others. Locally monotonic symmetric functors automatically preserve embedding-projection pairs, and we take advantage of this for finding recursively defined types.

So, building up a model of $\lambda_{2}$ in the sense of Seely, we take as global category, $\mathbf{E}$, that with 
the $\Omega^{m}$ as objects, where $\Omega$ is $\mathbf{R}^{D}$, and as morphisms from $\Omega^{m}$ to $\Omega^{n}$ the effective symmetric functors (these will interpret type expressions). This category has all finite products, and $\Omega^{m}$ is the $m$-fold product of $\Omega$. We write $\prod_{i}^{m}: \Omega^{m} \rightarrow \Omega$ $(0<i \leq m)$ for the projection functors.

For products and functional types we use effective, symmetric functors $\times$ and $\rightarrow$ defined on good pers as their product and function spaces, and by $\langle f, g\rangle \times\left\langle f^{\prime}, g^{\prime}\right\rangle=\left\langle f \times f^{\prime}, g \times g^{\prime}\right\rangle$ and by $\langle f, g\rangle \rightarrow\left\langle f^{\prime}, g^{\prime}\right\rangle=\left\langle g \rightarrow f^{\prime}, f \rightarrow g^{\prime}\right\rangle$ on morphisms, where on the right we are using the corresponding functors over Per.

For universal quantification, suppose we have $F: \Omega^{m+1} \rightarrow \Omega$, symmetric and realised by $\phi$. We define $\left(\forall_{m} F\right): \Omega^{m} \rightarrow \Omega$ on objects to be $\bigcap_{T} F(\hat{S}, T)$. As for morphisms, assume that $x_{i} \vdash h_{i}: S_{i} \rightarrow S_{i}^{\prime}$ in $\Omega$. Set $i p=\langle\lambda x . x, \lambda x . x\rangle$. This is an element of $D$ which realises every identity in $\Omega$. So we get an element $\phi(\hat{x}, i p)$ which realises $F\left(\hat{h}, i d_{T}\right): F(\hat{S}, T) \rightarrow F\left(\hat{S}^{\prime}, T\right)$ for every $T$ and so $\phi(\hat{x}, i p)$ realises a morphism from $\left(\forall_{m} F\right)(\hat{S})$ to $\left(\forall_{m} F\right)\left(\hat{S}^{\prime}\right)$ which we take to be $\left(\forall_{m} F\right)(\hat{h})$. (Unfortunately, bounded universal quantification does not seem to work. On objects, one would define $\left(\forall_{m}^{\subset} F\right): \Omega^{m+1} \rightarrow \Omega$ by: $\left(\forall_{m}^{\subset} F\right)(\hat{S}, T)=\bigcap_{T^{\prime} \subset T} F(\hat{S}, T)$; but there is no evident definition for morphisms.)

For recursive types, now suppose we have $F: \Omega^{m+1} \rightarrow \Omega$ realised symmetrically by $\phi$, and wish to define $\left(\mu_{m} F\right): \Omega^{m} \rightarrow \Omega$. First we define iterates $F^{(n)}: \Omega^{m} \rightarrow \Omega$ taking the constantly $\mathbb{1}$ functor for $F^{(0)}$, realised by $\phi^{(0)}=\lambda \hat{x} . \perp$ and taking $F^{(n+1)}=F \circ\left\langle\prod_{1}^{m}, \ldots, \prod_{m}^{m}, F^{(n)}\right\rangle$ realised by $\phi^{(n+1)}=\lambda \hat{x} \cdot \phi\left(\hat{x}, \phi^{(n)}(\hat{x})\right)$. Note that $\phi^{(n)} \sqsubseteq \phi^{(n+1)}$. Next natural transformations $\nu_{n n^{\prime}}: F^{(n)} \rightarrow F^{\left(n^{\prime}\right)}\left(n \leq n^{\prime}\right)$ can be defined by setting $\left(\nu_{0 n^{\prime}}\right)_{\hat{S}}$ to be $\langle\perp, \perp\rangle$ and $\left(\nu_{(n+1)\left(n^{\prime}+1\right)}\right)_{\hat{S}}=$ $F\left(i d_{\hat{S}},\left(\nu_{n n^{\prime}}\right) \hat{S}\right)$. Set $\bar{\nu}_{0}=\perp($ in $D)$ and $\bar{\nu}_{(n+1)}=$ $\phi\left(\hat{i p}, \bar{\nu}_{n}\right)$. Then $\bar{\nu}_{n}$ realises $\left(\nu_{n, n^{\prime}}\right)_{\hat{S}}$, for any $\hat{S}$ and $\bar{\nu}_{n}^{\star}=\bar{\nu}_{n} \sqsubseteq \bar{\nu}_{n+1}$.

This yields an $\omega$-cochain of embedding-projection pairs $\triangle_{\hat{S}}=\left\langle F^{(n)}(\hat{S}),\left(\nu_{n, n^{\prime}}\right){ }_{\hat{S}}\right\rangle$, as above with $\alpha_{n}=f s t\left(\bar{\nu}_{n}\right)$. We can therefore construct a colimiting cone $\rho_{\hat{S}}: \triangle \rightarrow\left(\mu_{m} F\right)(\hat{S})$ (defining the object part of $\left.\mu_{m} F\right)$ with $\left\langle\bar{\nu}_{n}\right\rangle$ realising $\rho_{\hat{S}}$. For the morphism part suppose $\hat{h}: \hat{S} \rightarrow \hat{S}^{\prime}$ then one takes $\left(\mu_{m} F\right)(\hat{h})=\bigvee\left(\rho_{\hat{S}^{\prime}}\right)_{n} \circ F^{(n)}(\hat{h}) \circ\left(\rho_{\hat{S}}\right)_{n}^{\dagger}$. This is realised by $\bigsqcup\left(\bar{\nu}_{n} \bullet \phi^{(n)}(\hat{x}) \bullet \bar{\nu}_{n}\right)$ (if $\left.x_{i} \vdash h_{i}\right)$ where $\bullet$ is the realiser for composition in $\mathbf{R}^{D}$; note this is a continuous function of $\hat{x}$.

Since $F$ is locally continuous one has that

$$
F\left(\hat{S}, \rho_{\hat{S}}\right): F(\hat{S}, \triangle) \rightarrow F\left(\hat{S},\left(\mu_{m} F\right) \hat{S}\right)
$$

is also colimiting. It follows from the "basic lemma" in [18] that the initial $F(\hat{S},-)$ algebra is $\left\langle\left(\mu_{m} F\right) \hat{S}, \alpha_{\hat{S}}\right\rangle$ where

$$
\alpha_{\hat{S}}: F\left(\hat{S},\left(\mu_{m} F\right) \hat{S}\right) \rightarrow\left(\mu_{m} F\right) \hat{S}
$$

is the mediating morphism from $F\left(\hat{S}, \rho_{\hat{S}}\right)$ to $\left(\rho_{\hat{S}}\right)^{-}$. This is just $\bigvee_{n \geq 0}\left(\rho_{\hat{S}}\right)_{n+1} \circ F\left(\hat{S},\left(\rho_{\hat{S}}\right)_{n}\right)^{\dagger}$. It has inverse $\alpha_{\hat{S}}^{\dagger}$, and $\bigsqcup_{n \geq 0}\left(\bar{\nu}_{n+1} \bullet \phi\left(\hat{i p}, \bar{\nu}_{n}\right)\right)$ realises it, independently of $\hat{S}$.

\section{Interpreting Terms}

To interpret terms we need an indexed category, $\mathbf{G}: \mathbf{E}^{o p} \rightarrow$ Cat. The objects of $\mathbf{G}\left(\Omega^{m}\right)$ have to be $\mathbf{E}\left(\Omega^{m}, \Omega\right)$, the symmetric effective functors. For the morphisms $\nu: F \rightarrow G$ we take the families $\nu_{\hat{S}}: F(\hat{S}) \rightarrow G(\hat{S})$ in $\mathbf{Q}$ such that there is a $\theta$ in $D$ which realises every $\nu_{\hat{S}}$ (we say $\theta$ realises $\nu$ ). For example, the first and second components of $\alpha_{S}$ (defined above) provide the morphisms needed to interpret the terms $\eta X: T p . T$ and $\eta^{-1} X: T p . T$.

Given an $H: \Omega^{n} \rightarrow \Omega^{m}$ in $\mathbf{E}$ we get a functor $\mathbf{G}(H): \mathbf{G}\left(\Omega^{m}\right) \rightarrow \mathbf{G}\left(\Omega^{n}\right)$ which acts by composition on the objects and if $\nu: F \rightarrow G$ in $\mathbf{G}\left(\Omega^{m}\right)$ then $\mathbf{G}(\nu): F \circ H \rightarrow G \circ H$ is $\nu_{H\left(\hat{S}^{\prime}\right)}$ at $\hat{S}^{\prime}$ in $\Omega^{n}$.

Each fibre is Cartesian closed, the structure being preserved on the nose. The terminal object in $\mathbf{G}\left(\Omega^{m}\right)$ is the "constantly $\mathbb{1}$ " functor $t^{m}: \Omega^{m} \rightarrow \Omega$. The unique $!^{m}: F \rightarrow t^{m}$ is that realised by $\lambda x . \perp$.

For binary products, given $F$ and $G$ in $\mathbf{G}\left(\Omega^{m}\right)$ their product is given by composition: $F \times{ }_{m} G=$ $(\times \circ\langle F, G\rangle)$. The projections are realised by $f s t$ and snd. If we have $\nu: H \rightarrow F$ and $\mu: H \rightarrow G$ (realised by $\theta$ and $\vartheta$ ) then their tuple $\langle\nu, \mu\rangle_{m}: H \rightarrow$ $F \times_{m} G$ is realised by $\lambda x .\langle\theta x, \vartheta x\rangle$.

The exponentiation of $F$ and $G$ is given by $F \rightarrow{ }_{m} G=(\rightarrow \circ\langle F, G\rangle)$. The evaluation morphism eval: $\left(F \rightarrow_{m} G\right) \times_{m} F \rightarrow G$ is realised by 
$\lambda x . f s t(x)(\operatorname{snd}(x))$. Given $\nu: H \times_{m} F \rightarrow G$, realised by $\theta, \operatorname{curry}(\nu): H \rightarrow\left(F \rightarrow_{m} G\right)$ is realised by $\lambda x \lambda y . \theta(\langle x, y\rangle)$.

For universal quantification, we need to describe $\forall$ as a functor $\forall: \mathbf{G}^{\Omega} \rightarrow \mathbf{G}$ of indexed categories, with $\forall$ right adjoint to the diagonal $\triangle: \mathbf{G} \rightarrow \mathbf{G}^{\Omega}$. Here $\mathbf{G}^{\Omega}$ is the indexed category $\mathbf{G}(-\times \Omega)$. We have to define for every $m \geq 0$ a functor $\forall_{m}: \mathbf{G}\left(\Omega^{m+1}\right) \rightarrow \mathbf{G}\left(\Omega^{m}\right)$. For objects $F: \Omega^{m+1} \rightarrow \Omega$ we can take $\forall_{m}(F)$ as above. For morphisms $\nu: F \rightarrow F^{\prime}$, realised by $\theta$, say, we have $\nu_{\hat{S}, T}: F(\hat{S}, T) \rightarrow F^{\prime}(\hat{S}, T)$ so then $\theta$ realises $\nu_{\hat{S}, T}$ for every $T$ and we take $\forall_{m}(\nu)_{\hat{S}}$ to be $[\theta]_{\left(\forall_{m} F\right) T,\left(\forall_{m} F^{\prime}\right) T}$.

Finally, to interpret term expressions for recursive elements, given $F$ and $G$ in $\mathbf{G}\left(\Omega^{m}\right)$ and $\nu: F \times_{m} G \rightarrow G$ we need $f i x(\nu): F \rightarrow G$. At $\hat{S}$ in $\Omega^{m}$ this is the least $g: F(\hat{S}) \rightarrow G(\hat{S})$ such that $g=\nu_{\hat{S}} \circ\left\langle i d_{F(\hat{S})}, g\right\rangle$. It is realised by $\lambda x . Y(\lambda y \cdot f(\langle x, y\rangle))$, where $f$ realises $\nu$ and $Y:(D \rightarrow D) \rightarrow D$ is the usual least fixed-point operator.

\section{The Metric-Space Approach}

Amadio introduced uniformity in order to solve type equations by the Banach fixed-point theorem. The method in fact allows one to give a Seely-style model of $\lambda_{2}$ extended with recursive types and elements, and it turns out that the closed types are interpreted as above; one can even go on to interpret bounded quantification. It is (still) not clear, however, how to extend this to $F \omega$. So, one defines a complete metric on the set $\mathcal{R}$ of good pers by: $d(S, T)=0$ if $S=T$, and $d(S, T)=2^{-c}$ if $c$ is the least natural number such that $\pi_{c}(S) \neq \pi_{c}(T)$. If $S_{n}$ is a Cauchy sequence its limit $S_{\infty}$ is given as in section 5: $x S_{\infty} y$ if $\forall n \forall_{\infty} m . \pi_{n} x S_{m} \pi_{n} y$.

For the Seely-style model take as global category, $\mathbf{M}$, that with the $\mathcal{R}^{m}$ as objects and as morphisms tuples $\left\langle F_{1}, \ldots, F_{n}\right\rangle: \mathcal{R}^{m} \rightarrow \mathcal{R}^{n}$ where each $F_{i}$ is either contractive or a projection. Take $\Omega$ to be $\mathcal{R}$. Both $\times$ and $\rightarrow$ act contractively on $\mathcal{R}$. Taking $\left({ }_{m} F\right): \Omega^{m} \rightarrow \Omega$ to be defined by $\left(\forall_{m} F\right)(\hat{S})=\cap_{T} F(\hat{S}, T)$, we obtain a contractive function if $F: \Omega^{m+1} \rightarrow \Omega$ is con- tractive or projects its last element, and a projection otherwise. Similarly, for recursive types $\left(\mu_{m} F\right): \Omega^{m} \rightarrow \Omega$, we define $F^{(n)}: \Omega^{m} \rightarrow \Omega$ on $\hat{S}$ as above. Then, in all cases, $F^{(n)}(\hat{S})$ is a Cauchy sequence, we take $\left(\mu_{m} F\right)(\hat{S})$ to be its limit, and find an actual equality: $F\left(\hat{S},\left(\mu_{m} F\right)(\hat{S})\right)=$ $\left(\mu_{m} F\right)(\hat{S})$.

For the indexed category $\mathbf{G}: \mathbf{M}^{o p} \rightarrow$ Cat we proceed as before taking the objects of $\mathbf{G}\left(\Omega^{m}\right)$ as $\mathbf{M}\left(\Omega^{m}, \Omega\right)$ and as morphisms $\nu: F \rightarrow G$ the families $\nu_{\hat{S}}: F(\hat{S}) \rightarrow G(\hat{S})$ in $\mathbf{Q}$ realised by a single element of $D$. In the case of recursive types, for example, one can interpret $\eta X: T p . T$ and $\eta^{-1} X: T p . T$ by the identity. Then the fibres are Cartesian closed, as before, and $\forall: \mathbf{G}^{\Omega} \rightarrow \mathbf{G}$ is also defined as before, as is $f i x(\nu)$.

In fact, this is essentially a re-presentation of our previous model: the interpretation of types is the object part of the previous one and the interpretation of terms is exactly the same. What we have gained is the knowledge that the morphisms used to interpret $\eta X: T p . T$ and $\eta^{-1} X: T p . T$ are identities and the recursive types are initial algebras.

To show all this, one, as it were, combines both models into a single one which also has contractiveness restrictions at the level of morphisms. (Most of the proofs are omitted.) First, for a good $S$, metricise $[S]$ by: $d_{S}\left([x]_{S},[y]_{S}\right)=$ $2^{-c\left([x]_{S},[y]_{S}\right)}$, where $c\left([x]_{S},[y]_{S}\right)$ is the least $n$ such that $\left[\pi_{n} x\right]_{S} \neq\left[\pi_{n} y\right]$ (and $\infty$ when $x=y$ ). This yields a complete ultra-metric (and it is compact and metricises the Lawson topology on $[S]$ see [11]). One can show that $f:[S] \rightarrow[T]$ is nonexpansive iff $\pi_{n}^{T} \circ f=\pi_{n}^{T} \circ f \circ \pi_{n}^{S}$ (where, for example, $\left.\pi_{n}^{S}\left([x]_{S}\right)=\left[\pi_{n} x\right]_{S}\right)$. One then takes the sup ultra-metric on functions from $[S]$ to $[T]$ by $d_{S, T}(f, g)=\sup _{x \in|S|} d_{T}\left(f\left([x]_{S}\right), g\left([x]_{S}\right)\right)$.

Now take EM to be the subcategory of $\mathbf{E}$ with the same objects, and as morphisms those functors $F: \Omega^{m} \rightarrow \Omega^{n}$ such that each $\left(\Pi_{i}^{n} \circ F\right)$ either is a projection, or is contractive in that it acts contractively on both objects and morphisms (see [2]); for $\mathbf{R}^{D}(S, T)$ we take $d\left(\langle f, g\rangle,\left\langle f^{\prime}, g^{\prime}\right\rangle\right)=$ $\max \left(d_{S, T}\left(f, f^{\prime}\right), d_{T, S}\left(g, g^{\prime}\right)\right)$. Then EM inherits its finite product structure from $\mathbf{E}$ and the effective symmetric functors $\times, \rightarrow: \Omega^{2} \rightarrow \Omega$ are 
also contractive. Further, universal quantification can be defined as in $\mathbf{E}$, as it preserves contractiveness.

The metric structure on morphisms is put to use when considering recursive types, $\left(\mu_{m} F\right)$ : $\Omega^{m} \rightarrow \Omega$, defined as before. As with $\mathbf{M}$ we find that $\left(\mu_{m} F\right)(\hat{S})$ is the limit of the Cauchy sequence $F^{(n)}(\hat{S})$ and obtain an actual equality $F\left(\hat{S},\left(\mu_{m} F\right)(\hat{S})\right)=\left(\mu_{m} F\right)(\hat{S})$, and we also get (as in [12]) that $\left(\mu_{m} F\right)$ acts contractively on objects. Now consider the action of $F(\hat{S},-)$ on $\Omega\left(F\left(\hat{S},\left(\mu_{m} F\right)(\hat{S})\right),\left(\mu_{m} F\right)(\hat{S})\right)$. One sees by the above equality that $i d_{\left(\mu_{m} F\right)(\hat{S})}$ is a fixedpoint. But one can show, by considering realisers, that $\alpha_{\hat{S}}$ is another fixed-point, and so $\alpha_{\hat{S}}$ is the identity by contractiveness. One has then to show that $\left(\mu_{m} F\right)$ acts contractively on morphisms. Finally, the indexed structure on EM is again inherited from $\mathbf{E}$ and the evident functors $\mathbf{E} \leftarrow \mathbf{E M} \rightarrow \mathbf{M}$ provide the needed relation between the three models.

\section{$9 \quad$ A Partial Preorder Model}

Good pers yield intrinsic partial orders with useful properties, which we have exploited in the construction of a model for $\lambda_{2}$, and which would also be relevant in giving a logic for $\lambda_{2}$. It is not necessary to start with pers, however: the same results can be obtained directly from preorders. In fact, the use of preorders is preferable in some respects, because it does not require meetclosure assumptions. Hence, preorder models are compatible with convex-powerdomain constructions on bifinite cpos. (There should be no difficulty, even in the original Scott-domain setting, in adding the Hoare and the Smyth powerdomains.)

Here we outline the main features of a model based on preorders. We drop assumption 4 (and no longer require that $D$ be a Scott domain, but only a bifinite cpo). A binary relation $S$ on $D$ is a partial preorder (ppo) if it is a preorder on $|S|=\{a \mid a S a\}$. It is a good partial preorder if it satisfies the axioms of completeness, uniformity, and if it extends the underlying partial order $\sqsubseteq$ (on $|S|$ ):
Extensiveness If $a, b \in|S|$ and $a \sqsubseteq b$ then $a S b$.

We do not require antisymmetry, convexity, or meet-closure properties.

Naturally, a partial equivalence relation $\simeq_{S}$ is associated with every $S: a \simeq_{S} b$ iff $a S b$ and $b S a$. Furthermore, if $S$ is good then $\simeq_{S}$ is complete and uniform. However, the intrinsic order obtained from $\simeq_{S}$ is not necessarily identical to $S$, but possibly stronger. It is identical to $S$ when $S$ is meet-closed. In the other direction, if $S$ is a per then $\leq_{S}$ is a complete, extensive partial preorder; it is uniform if $S$ is.

Let $S$ be a ppo. We write $[x]_{S}$ for the equivalence class under $\simeq_{S}$ of any $x$ in $|S|$; we write $[S]$ for the set of such, partially ordered by: $[x]_{S} \leq[y]_{S}$ iff $x S y$. We define $f: T^{S}$ as for pers. The category Ppo has the ppos as objects and as morphisms the monotonic $g:[S] \rightarrow[T]$ such that for some $f: T^{S}$ and for all $x$ in $|S|$, $g\left([x]_{S}\right)=[f(x)]_{T}$. We write $[f]_{S, T}$ for $g$ and also $f \vdash g(f$ realises $g)$. Ppo is effective in the same sense and the same way as Per. The analogue of Theorem 1 holds for a good ppo $S$, that is, $[S]$ is an $\omega$-algebraic cpo. (Actually it is even bifinite as $\pi_{n}^{S}:[x]_{S} \mapsto\left[\pi_{n} x\right]_{S}$ is an increasing sequence of projections with finite range and lub the identity.) The intrinsic topology is defined, as before; it is the Scott topology (for good ppos) and so Ppo morphisms between good ppos are Scott continuous.

We have $\mathbb{D}$ and $\mathbb{I N}$ as good ppos. Ppo is Cartesian closed with products and function spaces defined as before (and now the analogue of Proposition 2 is trivial for any ppo). These constructions preserve each of extensiveness, completeness, and uniformity. The intersection of a family of ppos is also one, and it is extensive (respectively, complete, uniform) if each ppo in the family is. Ordering the morphisms of Ppo pointwise we get an isomorphism $\phi:\left[R^{S}\right] \cong$ $\mathbf{P p o}(R, S)$. The full subcategory $\mathbf{p Q}$ of good ppos is then an $\mathbf{O}$-category; we take $\mathbf{p R}$ to be the further subcategory of the uniform morphisms (as defined before). Colimits in $\mathbf{p} \mathbf{R}^{D}$ work out as before, and then so too does the definition of a Seely model allowing the interpretation of 
recursive elements and types. The material on metric spaces should also extend, but we have not investigated this.

Turning to the powerdomains, we add to assumption 2 the existence of a strict retraction pair, $\mathcal{P}(D) \stackrel{e_{p}}{\longrightarrow} D \stackrel{r_{p}}{\longrightarrow} \mathcal{P}(D)$, and to assumption 3 that $\pi_{n+1}\left(e_{p}(X)\right)=e_{p}\left(\left(\pi_{n} X\right)^{*}\right)$ (in the notation of [14] $-Y^{*}$ is the least convex Lawsonclosed set containing $Y$ ). We identify any $X$ in $\mathcal{P}(D)$ and $e_{p}(X)$. Restricting, for brevity, to good ppos $R$, we define the "power-ppo" by:

$$
\begin{aligned}
& \mathcal{P}(R)=\left\{\langle X, Y\rangle \in \mathcal{P}(D)^{2} \mid\right. \\
& (\forall x \in X \exists y \in Y . x R y) \wedge(\forall y \in Y \exists x \in X . x R y)\}
\end{aligned}
$$

obtaining a good ppo. Useful lemmas in showing this are that each $\pi_{n}$ is Lawson continuous and good ppos are closed under limits in the Lawson topology.

There are many associated functions. The union function on $\mathcal{P}(D)$ can be considered over $D$ by using the retractions and for any $R$ it realises a binary morphism over $\mathcal{P}(R)$ turning it into a semilattice; however, this does not seem to be the free such semilattice (as in [10]) and there may not be one. The other associated functions can be organised as a strong monad, following Moggi [13]. The unit is realised by the singleton function in $D \rightarrow \mathcal{P}(D)$, the multiplication is realised by the "big union" in $\mathcal{P}^{2}(D) \rightarrow \mathcal{P}(D)$, and the strength is realised by $t: D \times \mathcal{P}(D) \rightarrow$ $\mathcal{P}(D \times D)$ where $t(x, Y)=\{x\} \times Y$.

There arises the question of the appropriate extension of $\lambda_{2}$. One course is to add a "powertype operator" to obtain types $\mathcal{P}(T)$ together with polymorphic constants for the associated functions. One can then ask how such a language can be given an operational semantics (for which our model is adequate) and so can be considered as a programming language; the powertypes present some difficulties.

\section{Acknowledgements}

We have benefited from comments from L. Cardelli and P.-L. Curien.

\section{References}

[1] R.M. Amadio, 1989. Recursion over realizability structures. To appear in Information and Computation.

[2] P. America and J.J.M.M. Rutten, 1988. Solving reflexive domain equations in a category of complete metric spaces. Proc. Third Workshop on the Mathematical Foundations of Programming Language Semantics, Springer-Verlag Lecture Notes in Computer Science 298, pp. 254-288. To appear in JCSS.

[3] A. Asperti and S. Martini, 1989. Categorical models of polymorphism. To appear.

[4] L. Cardelli and G. Longo, 1990. A semantic basis for Quest. Research Report No. 55, Systems Research Center, Digital Equipment Corporation. To appear in the Proceedings of the 1990 ACM Conference on Lisp and Functional Programming.

[5] F. Cardone, 1989. Relational semantics for recursive types and bounded quantification. ICALP 1989, Springer-Verlag Lecture Notes in Computer Science 372, pp. 164-178.

[6] T. Coquand, C.A. Gunter, and G. Winskel, 1987. dI-domains as a model of polymorphism. Proc. Third Workshop on the Mathematical Foundations of Programming Language Semantics, Springer-Verlag Lecture Notes in Computer Science 298, pp. 344363.

[7] T. Coquand, C.A. Gunter, and G. Winskel, 1989. Domain theoretic models of polymorphism. Information and Computation, 81, 2:123-167.

[8] J.-Y. Girard, 1972. Interprétation fonctionelle et élimination des coupures dans l'arithmétique d'order supérieur. Thèse de Doctorat d'Etat, Paris.

[9] C.A. Gunter and D.S. Scott, 1989. Semantic domains. To appear in Handbook of Theoretical Computer Science, eds. A.R. Meyer, 
M. Nivat, M.S. Paterson, and D. Perrin. North Holland.

[10] M. Hennessy and G.D. Plotkin, 1979. Full abstraction for a simple parallel programming language. Mathematical Foundations of Computer Science, Springer-Verlag Lecture Notes in Computer Science 74, pp. 108120 .

[11] P.T. Johnstone, 1982. Stone spaces. Cambridge University Press.

[12] D. MacQueen, G.D. Plotkin, and R. Sethi, 1986. An ideal model for recursive polymorphic types. Information and Control, 71:95130.

[13] E. Moggi, 1989. Computational lambdacalculus and monads. Proc. Fourth LICS, pp. $14-23$.

[14] G.D. Plotkin, 1976. A powerdomain construction. Siam J. Comput. 5:452-487.

[15] G. Rosolini, 1986. Continuity and effectiveness in topoi. D.Phil. thesis, University of Oxford.

[16] D.S. Scott, 1976. Data types as lattices. Siam J. Comput. 5:522-587.

[17] R.A.G. Seely, 1987. Categorical semantics for higher-order polymorphic lambda calculus. Journal of Symbolic Logic, 52:4.

[18] M.B. Smyth and G.D. Plotkin, 1982. The category-theoretic solution of recursive domain equations. Siam J. Comput. 11:761783.

[19] A.S. Troelstra, 1973. Metamathematical investigations of intuitionistic arithmetic and analysis. Springer-Verlag Lecture Notes in Mathematics 344. 